\title{
Speciation and the evolution of dispersal along environmental gradients
}

Simone K. Heinz (simone.heinz@fa.uib.no)

Rupert Mazzucco (mazzucco@iiasa.ac.at)

Ulf Dieckmann (dieckmann@iiasa.ac.at)

\section{Approved by \\ Detlof Von Winterfeldt \\ Director}

July 2011 Institute, its National Member Organizations, or other organizations supporting the work. 


\title{
Speciation and the evolution of dispersal along environmental gradients
}

\author{
Simone K. Heinz ${ }^{1}$, Rupert Mazzucco² \& Ulf Dieckmann ${ }^{2,3}$ \\ ${ }^{1}$ Department of Biology, University of Bergen, P.O. Box 7800, 5020 Bergen, Norway \\ ${ }^{2}$ Evolution and Ecology Program, International Institute for Applied Systems Analysis, \\ Schlossplatz 1, 2361 Laxenburg, Austria \\ ${ }^{3}$ Section Theoretical Biology, Institute of Biology, Leiden University, Kaiserstraat 63, \\ 2311 GP Leiden, The Netherlands
}

\begin{abstract}
We analyze the joint evolution of an ecological character and of dispersal distance in asexual and sexual populations inhabiting an environmental gradient. Several interesting phenomena resulting from the evolutionary interplay of these characters are revealed. First, asexual and sexual populations exhibit two analogous evolutionary regimes, in which either speciation in the ecological character occurs in conjunction with evolution of short-range dispersal, or dispersal distance remains high and speciation does not occur. Second, transitions between these two regimes qualitatively differ between asexual and sexual populations, with the former showing speciation with long-range dispersal and the latter showing no speciation with short-range dispersal. Third, a phenotypic gradient following the environmental gradient occurs only in the last case, i.e., for non-speciating sexual populations evolving towards short-range dispersal. Fourth, the transition between the evolutionary regimes of long-range dispersal with no speciation and short-range dispersal with speciation is typically abrupt, mediated by a positive feedback between incipient speciation and the evolution of short-range dispersal. Fifth, even though the model of sexual evolution analyzed here does not permit assortative mating preferences, speciation occurs for a surprisingly wide range of conditions. This illustrates that dispersal evolution is a powerful alternative to preference evolution in enabling spatially distributed sexual populations to respond to frequency-dependent disruptive selection.
\end{abstract}

\section{Introduction}

The study of speciation is an active area of research in evolutionary biology and is stimulating wide-ranging discussions (see Turelli at al. 2001 for a review). Mounting empirical 
evidence suggests that sympatric speciation can occur in nature (e.g., Schliewen et al. 1994; Bush and Smith 1998; Filchack et al. 2000; Wilson et al. 2000; Schliewen et al. 2001). This has spawned corresponding theoretical research striving to identify key mechanisms that may promote such speciation (e.g., Maynard Smith 1966; Turner and Burrows 1995; Kawecki 1997; Kondrashov and Kondrashov 1999; Higashi et al. 1999; Dieckmann and Doebeli 1999; Drossel and Kane 2000; Doebeli and Dieckmann 2003; see also the review by Via 2001). In particular, resource competition leading to frequency-dependent disruptive selection has received wide recognition as a potential ecological driver of sympatric speciation (MacArthur and Levins 1967; Rosenzweig 1978; Christiansen and Loeschcke 1980; Slatkin 1980; Seger 1985; Taper and Case 1985; Maynard Smith and Brown 1986; Abrams et al. 1993; Vincent et al. 1993; Doebeli 1996a, 1996b; Metz et al. 1996; Law et al. 1997; Meszéna et al. 1997; Geritz et al. 1998; Dieckmann and Doebeli 1999; Geritz et al. 1999; Jansen and Mulder 1999; Kisdi 1999; Kondrashov and Kondrashov 1999; Day 2000; Doebeli and Dieckmann 2000; Drossel and McKane 2000; Kisdi 2001; Kisdi and Geritz 2001; Kisdi et al. 2001; Schreiber and Tobiason 2003; Egas et al. 2004, 2005; Doebeli et al. 2007; Ito and Dieckmann 2007).

Related research has shown that parapatric speciation driven by frequency-dependent competition can be promoted by environmental gradients (Doebeli and Dieckmann 2003; Mizera and Meszéna 2003; Leimar et al. 2008). This facilitation results from a gradientinduced type of frequency-dependent selection: local competition and local adaptation along an environmental gradient imply phenotype-dependent competition and thus frequency-dependent selection (Doebeli and Dieckmann 2003). Local adaptation is thus a prerequisite for gradient-induced frequency-dependent selection and, obviously, can be impeded by frequent or long-range dispersal. This means that the potential for parapatric speciation driven by gradient-induced frequency-dependent selection is bound to depend on the dispersal rates and distances of individuals: for relatively low mobility, speciation was found to occur readily, whereas increasing mobility first reduces and eventually extinguishes gradient-induced speciation (Doebeli and Dieckmann 2003).

It is important to realize that the mobilities for which speciation through gradientinduced frequency-dependent selection can occur are too large to imply isolation by distance (Doebeli and Dieckmann 2003). In general, speciation processes in sexual populations are hindered by the mixing of gene pools through segregation and recombination (e.g., Felsenstein 1981). In parapatric speciation, this obstacle may be overcome by assortative mating preferences (Doebeli and Dieckmann 2003) or by isolation by distance (Wright 1943). The traditional notion of isolation by distance, which is applicable when mobilities are very low compared to the spatial distances between subpopulations, thus 
highlights a qualitatively different mechanism by which gradients may facilitate diversification: gene pools of low-mobility ecotypes favored by local selection along environmental gradients (Turesson 1922) are genetically segregated from each other to an extent that allows processes of local adaptation, and potentially also of speciation, to run their course relatively unhindered (e.g., Slatkin 1973; Turelli et al. 2001).

Decreasing a population's mobility thus has a twofold effect on the potential for parapatric speciation: a decrease of mobility below a relatively high threshold enables speciation through gradient-induced frequency-dependent selection and the resultant evolution of assortative mating preferences, while a decrease to a much lower level enables speciation through isolation by distance. This realization puts a spotlight on the evolution of dispersal in parapatric speciation.

In more general contexts, many model-based analyses have investigated the evolution of dispersal rates (McPeek and Holt 1992; Holt and McPeek 1996; Olivieri et al. 1995; Doebeli and Ruxton 1997; Gandon 1999; Ronce et al. 2000, 2005; Mathias et al. 2001; Poethke and Hovestadt 2002; Cadet et al. 2003; Parvinen et al. 2003; Kisdi 2004), dispersal distances (Ezoe 1998; Savill and Hogeweg 1998; Hovestadt et al. 2001; Murrell et al. 2002), and, more recently, directedness of dispersal (Armsworth and Roughgarden 2005; Heinz and Strand 2006). Selection has been shown to favor dispersal through mechanisms including inbreeding avoidance (Bengtsson 1978; Waser 1986; Motro 1991; Gandon 1999) and kin competition (Hamilton and May 1977; Frank 1986; Taylor 1988; Gandon and Michalakis 1999; Rousset and Gandon 2002).

Doebeli and Dieckmann (2003) investigated the implications of mobility for parapatric speciation through gradient-induced frequency-dependent selection by assuming different, but fixed, levels of mobility. This naturally begs the question how such mobility would adapt if it were subject to evolution. Classical trade-offs affecting dispersal evolution of course also apply to the model by Doebeli and Dieckmann (2003). On the one hand, competition in this model is higher between individuals with similar phenotypes than for dissimilar individuals, favoring offspring that move away from their parents. On the other hand, the change of environmental conditions along a sufficiently steep gradient is bound to favor low mobility. The balance between these two opposing forces of selection might thus be expected to cause the evolution of intermediate mobility. Such simple qualitative reasoning, however, ignores the intricacies resulting from the dynamic interplay between speciation and dispersal evolution. As explained above, the potential for parapatric speciation is certainly influenced by dispersal, but also the converse applies: the evolution of dispersal is expected to be influenced by the distribution of phenotypes around and along an environmental gradient, and thus by speciation. 
Here we analyze the joint evolution of an ecological character and population mobility in asexual and sexual populations inhabiting an environmental gradient. We thus extend the parapatric speciation model of Doebeli and Dieckmann (2003) by letting dispersal and mating distances evolve, instead of using fixed values. Of the three dimensionless parameters of this model identified by Doebeli and Dieckmann (2003), two parameters (the scaled slope of the environmental gradient and the scaled width of the phenotypic competition function) were systematically varied, while the third parameter (the scaled dispersal distance) was allowed to evolve. In this way, our results below offer an exhaustive overview of the interplay between the evolution of local adaptation and mobility in a simple model of parapatric speciation, by showing which dispersal and mating distances evolve under different conditions and by examining the circumstances under which speciation can occur.

\section{Model Description}

The structure of the model description below is inspired by suggestions for describing individual- and agent-based models in a standardized manner (Grimm and Railsback 2005; Grimm et al. 2005, 2006).

\section{Purpose}

The purpose of the model is to understand the interplay between speciation and dispersal evolution along environmental gradients. The model extends the one by Doebeli and Dieckmann (2003) by incorporating the evolution of dispersal distances, and, in the case of sexual reproduction, of mating distances. We consider either asexual or sexual reproduction, without, however, ever incorporating assortative mating preferences. The resultant model operates in continuous space and time and provides an individual-based, stochastic, and spatially explicit description of phenotypic evolution.

\section{Environment and state variables}

The environment considered in the model is two-dimensional and continuous, with spatial locations identified by coordinates $0 \leq x, y \leq 1$. An environmental gradient exists in the $x$ direction, while the $y$-direction is ecologically neutral. Individuals living in this environment differ by location and phenotype. Phenotypes are denoted by $u$ and $v$, where $u$ is the ecological character affecting local adaptation along the environmental gradient and $v$ is the dispersal character affecting distances of natal dispersal. In the sexual model, an additional mating character $w$ affects the distance of mate searching. At any moment in time, the state of the system is fully given by the state $\left(x_{i}, y_{i}, u_{i}, v_{i}\right)$ or $\left(x_{i}, y_{i}, u_{i}, v_{i}, w_{i}\right)$, respectively, of all individuals $i=1, \ldots, N$, where $N$ is the current number of individuals. 


\section{Environmental gradient}

The carrying capacity density for the ecological phenotype $u$ at spatial location $(x, y)$ is $K(u, x, y)=K_{0} \cdot N_{\sigma_{K}}\left(u-u_{0}(x)\right)$, where $N_{\sigma}(z)=\exp \left(-\frac{1}{2} z^{2} / \sigma^{2}\right)$ denotes a normal, or Gaussian, function, and $u_{0}(x)=g \cdot\left(x-\frac{1}{2}\right)+\frac{1}{2}$ describes which ecological phenotype maximizes $K$ at location $x$. This implies an environmental gradient with slope $g$. Along this gradient, the phenotypic range around $u_{0}(x)$ that offers high carrying capacity has a width of $\sigma_{K}$.

\section{Boundary conditions}

Boundaries in the ecologically neutral $y$-direction are chosen to be periodic. Owing to the environmental gradient, environmental conditions differ at locations $x=0$ and $x=1$, so that periodic boundary conditions in the $x$-direction are not biologically meaningful. We thus investigate two other kinds of boundary conditions in the $x$-direction: impermeable and cline-periodic. Impermeable boundaries imply that values $x<0$ (or $x>1$ ) are replaced with values 0 (or 1, respectively). Cline-periodic boundary conditions (Leimar et al. 2008) imply that values $x<0$ (or $x>1$ ) are replaced with values $x+1$ (or $x-1$, respectively), while at the same time ecological phenotypes are replaced with values $u+g$ (or $u-g$, respectively). The latter ensures that the carrying capacity density experienced by individuals is unaffected by the imposed relocation. Cline-periodic boundary conditions are thus similar to spatially periodic boundary conditions, except for the additional correction of the ecological phenotype $u$.

\section{Phenotype ranges and initialization}

Initially, individuals are distributed randomly across the two-dimensional environment, with uniform probability density. For impermeable boundary conditions, the ecological characters of all individuals are initially set to $u_{i}=0.5$ and the dispersal characters are set to $v_{i}=0.5$; the mating characters, where applicable, are set to $w_{i}=0.2$. All characters are then kept in the range $0 \leq u, v, w \leq 1$. For cline-periodic boundary conditions, the initial ecological characters $u_{i}$ are randomly drawn from the range $0 \leq u \leq 1$ with uniform probability density, and are subsequently left unbounded.

\section{Process overview and scheduling}

Individuals undergo a continuous-time birth-death process, so that generations are overlapping. The current system state determines the birth and death rates, $b_{i}$ and $d_{i}$, of all individuals. On this basis, the population-level birth, death, and event rates are obtained as $B=\sum_{i=1}^{N} b_{i}, D=\sum_{i=1}^{N} d_{i}$, and $E=B+D$, respectively. The time lapse until the next event 
is drawn from an exponential distribution with mean $1 / E$. After the type of event is chosen according to probabilities $B / E$ and $D / E$, the affected individual is chosen according to probabilities $b_{i} / B$ or $d_{i} / D$. According to the chosen event type, the chosen individual either reproduces or dies.

\section{Interactions}

Individuals interact through local resource competition affecting their death rates. Individuals that are far apart either in space or in ecological phenotype interact less strongly than individuals that are spatially close or ecologically similar. When reproduction is sexual, individuals also interact by locally choosing a mating partner for producing offspring. Through these interactions, the fitness $b_{i}-d_{i}$ of individual $i$ is locally frequencydependent, as it varies with the distribution of phenotypes in the vicinity of individual $i$. In the case of periodic ( $y$-direction) and cline-periodic ( $x$-direction) boundary conditions, interactions stretch across boundaries.

\section{Stochasticity}

The model comprises several types of stochasticity: demographic stochasticity, affecting time lapses between events, as well as the sequence of events; dispersal stochasticity, affecting the direction and distance of natal dispersal; and mutation stochasticity, affecting the direction and distance of phenotypic changes resulting from mutation. When reproduction is sexual, these are complemented by two further types of stochasticity: mating stochasticity, affecting the choice of mating partner in an individual's vicinity; and segregation-recombination stochasticity, affecting the phenotypes of offspring depending on those of their parents.

\section{Death events}

The death rate of individual $i$ is $d_{i}=n_{\text {eff }}\left(u_{i}, x_{i}, y_{i}\right) / K\left(u_{i}, x_{i}, y_{i}\right)$, where $n_{\text {eff }}\left(u_{i}, x_{i}, y_{i}\right)$ is the effective number of individuals with which individual $i$ is competing,

$$
n_{\text {eff }}\left(u_{i}, x_{i}, y_{i}\right)=\left(2 \pi \sigma_{s}^{2}\right)^{-1} \sum_{j=1, j \neq i}^{N} N_{\sigma_{c}}\left(u_{j}-u_{i}\right) N_{\sigma_{s}}\left(x_{j}-x_{i}\right) N_{\sigma_{s}}\left(y_{j}-y_{i}\right) .
$$

This means that individuals compete the less strongly the more their spatial coordinates and ecological phenotypes differ, with the attenuation of competition strength being described by normal functions with standard deviations $\sigma_{c}$ (the width of the phenotypic competition function scaling the effect of $u$ ) and $\sigma_{s}$ (the width of the spatial competition function scaling the effects of $x$ and $y$ ). The normalization factor $\left(2 \pi \sigma_{s}^{2}\right)^{-1}$ ensures that $n_{\text {eff }}$ is 
independent of $\sigma_{s}$ in a spatially uniform population. Upon its death, the individual is removed from the population.

\section{Birth events and dispersal}

The birth rates of individuals are constant, $b_{i}=b$. Upon its birth, the new individual is inserted into the population. Its spatial coordinates $x$ and $y$ are drawn from normal distributions with means equal to values $x_{i}$ and $y_{i}$ of the focal parent, and with standard deviations $v_{i}$ reflecting the effect of natal dispersal.

When reproduction is sexual, the focal parent $i$ randomly chooses a mating partner $j$, with mating weights given by $N_{w_{i}}\left(x_{j}-x_{i}\right) N_{w_{i}}\left(y_{j}-y_{i}\right)$, where $w_{i}$ is the mating distance of individual $i$. The choice of partner thus depends on spatial distance alone, so that mating preferences are never assortative with regard to phenotype. There is no direct cost associated with having a high mating distance $w_{i}$ and there is also no direct cost of dispersal. The offspring's phenotypes $u, v$, and $w$ are drawn from normal probability distributions with means equal to mid-parental values $\frac{1}{2}\left(u_{i}+u_{j}\right), \frac{1}{2}\left(v_{i}+v_{j}\right), \frac{1}{2}\left(w_{i}+w_{j}\right)$ and with standard deviations $\frac{1}{2}\left|u_{i}-u_{j}\right|, \frac{1}{2}\left|v_{i}-v_{j}\right|, \frac{1}{2}\left|w_{i}-w_{j}\right|$, reflecting the effects of segregation and recombination. The expressions used for the standard deviations ensure that normal distributions of parental phenotypes are invariant under segregation and recombination. (Some other studies used population-level averages to specify these standard deviations, usually setting them equal to a quarter of the population-level standard deviation in the character considered: such an approach, however, is nonsensical when populations, as is the case here, are not panmictic.)

When reproduction is asexual, the phenotypes $u=u_{i}$ and $v=v_{i}$ are faithfully inherited from parent to offspring (up to mutation, see next paragraph).

Finally, the offspring's phenotypes $u, v$, and $w$ may be displaced with probability $\mu_{m}$ by random increments drawn from a normal distribution with mean 0 and standard deviation $\sigma_{m}$, reflecting the effect of mutation.

\section{Observables}

For model testing, it is helpful to observe the spatio-phenotypic distribution of individuals. For model analysis, the marginal phenotypic distributions of ecological character, dispersal character, and mating character are recorded.

Speciation is identified as follows. For asexual populations, we require the initially unimodal phenotype distribution to branch into a visibly bi- or multimodal distribution (Fig. 5a,b). For sexual populations, we additionally require that not more than a very few hybrids 
be present between candidate branches, implying sharp modes of the bi- or multimodal phenotype distribution (Fig. 5d).

\section{Parameters}

A list of default parameter values is provided in Table 1. Doebeli and Dieckmann (2003) showed that the asexual model defined above has no more than three essential parameters: the scaled width of the phenotypic competition function, $c=\sigma_{c} / \sigma_{K}$, the scaled slope of the environmental gradient, $s=g \sigma_{s} / \sigma_{K}$, and the scaled dispersal distance, $v / \sigma_{s}$. In our analyses below, we systematically vary the two dimensionless parameters $c$ and $s$, while allowing the dispersal distances $v$, and in the sexual model, the mating distances $w$, to evolve.

\section{Results}

Results below are first presented for asexual populations, thus offering a helpful baseline for the subsequent investigation of evolution in sexual populations.

\section{Asexual populations}

When reproduction is asexual, the joint evolution of the ecological phenotype and the dispersal phenotype results in three qualitatively different evolutionary outcomes: (i) speciation with short-range dispersal (Fig. 5a), (ii) speciation with long-range dispersal (Fig. 5b), and (iii) no speciation with long-range dispersal (Fig 5c).

These outcomes occur in three sharply delineated regions of the model's parameter space, spanned by the scaled slope $s$ of the environmental gradient and by the scaled width $c$ of the phenotypic competition function (Fig. 1, for impermeable boundary conditions in the $x$-direction; see Table 2 for an overview). First is a large parameter region on the righthand side of Fig. 1a, in which speciation occurs in conjunction with the evolution of shortrange dispersal. The existence of this region is in line with results by Doebeli and Dieckmann (2003) showing that for $c \geq 1$ speciation occurs for a larger range of mobilities when gradients are steep (as long as $s \leq 1$ ). Second is a parameter region in the upper left corner of Fig. 1a, in which dispersal distance remains high without speciation. This is of course facilitated by shallow environmental gradients. That speciation does not occur here is in line with results by Dieckmann and Doebeli (2003) showing that speciation is not expected for $c \geq 1$ unless facilitated by a sufficiently steep environmental gradient and accompanied by sufficiently low mobility. Third is a parameter region in the lower left corner of Fig. 1a, in which speciation occurs in conjunction with the evolution of long-range dispersal. Again, the latter evolution is promoted by the shallowness of the environmental gradient in this 
region, and speciation is expected since $c<1$. Very similar results are obtained when clineperiodic boundary conditions are used for the $x$-direction (Fig. 2). Here, the shift of ecological phenotypes by $g$ when individuals traverse the $x$-boundaries appears to facilitate the evolution of short-range dispersal.

Of the three identified regions, the upper part of the first region is perhaps most remarkable ( $c \geq 1$, and $s \leq 1$ but large). In this part, speciation cannot occur in the absence of a gradient $(s=0)$, so that all speciation encountered here is strictly gradient-induced. Doebeli and Dieckmann (2003) showed that under such conditions speciation can only occur if mobility is sufficiently low. Dispersal evolution takes care of this, so that the latter proviso disappears.

\section{Abrupt transition between long-range and short-range dispersal}

What is surprising is the abrupt nature of the transition between evolutionary outcomes with long-range and short-range dispersal. Plotting the evolved dispersal distance as a function of the environmental gradient's slope (Fig. 1b) shows that a gradually decreasing slope $s$ triggers an abrupt increase in the evolved dispersal distance $v$, especially for higher values of $c$.

The mere fact that dispersal distance increases when $s$ is decreased is easily understood. The cost of dispersal - resulting from the likelihood of reaching a location to which the dispersing individual is less adapted - decreases with $s$. This means that the benefit of dispersal - resulting from the likelihood of reaching a location that is less crowded and at which competition is thus diminished - prevails when $s$ is low.

But how can the observed threshold effect be explained? Speciation through gradientinduced frequency-dependent selection has been shown to occur more easily when mobility is low (Doebeli and Dieckmann 2003). Short-range dispersal thus facilitates speciation. Interestingly, also the converse is true: speciation may facilitate the evolution of short-range dispersal. Once a unimodal distribution of phenotypes has split into several phenotypic clusters along the environmental gradient, dispersal becomes less favorable, as it likely implies moving to locations where the dispersing individual has to compete with other, betteradapted phenotypes. Therefore, short-range dispersal not only facilitates speciation, but the onset of speciation also facilitates the evolution of short-range dispersal. This positive feedback between dispersal evolution and speciation causes a threshold effect in the evolution of dispersal distances. When this feedback loop is disrupted - because there is no incipient speciation, or because selection does not favor short-range dispersal - evolved dispersal distances suddenly increase. This explains why the transition between short-range and long-range dispersal is so sharp for $c \geq 1$, since here speciation is contingent on low 
mobility. For $c<1$, speciation can occur independent of mobility, so that the mutual dependence between speciation and dispersal evolution becomes one-sided instead, resulting in a more gradual transition. This effect also explains the shift of the regime boundary in the cline-periodic case (Fig. 2).

\section{Sexual populations}

Also when reproduction is sexual, evolution results in three qualitatively different evolutionary outcomes (Figs. 3, 4, 5d,e,f; see Table 2 for an overview): (i) speciation with shortrange dispersal (upper right corner of Figs. 3a, 4a; Fig. 5d), (ii) no speciation with shortrange dispersal (lower right corner of Figs. 3a, 4a; Fig. 5e), and (iii) no speciation with long-range dispersal (left-hand side of Figs. 3a, 4a; Fig. 5f). While cases (i) and (iii) both occur under asexual as well as under sexual evolution, case (ii) for sexual evolution (no speciation, short-range dispersal) and case (ii) for asexual evolution (speciation, long-range dispersal) characterize diametrically opposite evolutionary outcomes.

For sexual evolution, speciation occurs when $c$ and $s \leq 1$ are both sufficiently large, while short-range dispersal evolves when $s \leq 1$ is sufficiently large. It is interesting to highlight that speciation in this model thus requires sufficiently large values of $c$. By contrast, speciation in non-spatial models of sexual evolution, based on the evolution of assortative mating, is facilitated by small values of $c<1$ (Dieckmann and Doebeli 1999). Figs. 3 and 4 also show that, as expected, speciation under sexual evolution cannot occur in conjunction with long-range dispersal: in the absence of potential for the evolution of assortative mating preferences, sexual parapatric speciation is contingent on sufficiently low mobility.

A more detailed comparison with the outcomes of asexual evolution yields the following observations. First, the parameter region corresponding to case (i) under sexual evolution is a bit smaller, and shifted towards higher values for $c$ and $s$, as compared to asexual evolution. Second, the parameter region corresponding to case (iii) is larger and extends towards all lower values of $c$. And third, as was already emphasized above, the transition between cases (i) and (iii) is mediated by an entirely different class of cases (ii). It is worth emphasizing that under sexual evolution it is only case (ii) that implies a phenotypic gradient following the environmental gradient.

As in the asexual model, we found a threshold effect for how evolved dispersal distances depend on the gradient's slope (Figs. 3b, 4b). This threshold effect is again explained by the interplay between speciation and dispersal evolution. In contrast to the asexual case, however, speciation of sexual populations requires short-range dispersal even for $c<1$. The mutual dependence between speciation and dispersal evolution thus extends across all val- 
ues of $c$. Accordingly, the transition of evolved dispersal distances remains relatively sharp along the entire boundary of the parameter region enabling speciation.

The evolution of mating distances with increasing slope of the environmental gradient at first follows the evolution of the dispersal distance. It is only after the onset of speciation that mating distances no longer seem to be driven to particularly small values. To explain this, we have to appreciate that the evolution of short-range dispersal in conjunction with speciation amounts to the formation of spatially segregated phenotypic clusters. While such clusters can only form if the mating distance is sufficiently small, merely searching for a mate outside an individual's own cluster will not lead to any significant production of hybrids when distances between adjacent clusters are so large that actual matings across cluster boundaries are very rare. This understanding is corroborated by our finding that small mating distances are selected for much more strongly when competition widths, and hence distances between adjacent clusters, are small (Fig. 3b, 4b; bottom panels).

In summary, the propensity for speciation in this model of sexual parapatric speciation is shaped by two key mechanisms: first, frequency-dependent selection is induced by the joint effect of local competition and local adaptation along the environmental gradient (Doebeli and Dieckmann 2003), and second, a positive feedback exists between incipient speciation and the evolution of short-range dispersal (as explained above).

\section{Discussion}

In this study we investigated the joint evolution of an ecological character and of dispersal distance in asexual and sexual populations inhabiting an environmental gradient. We identified several interesting phenomena resulting from the evolutionary interplay of these characters.

Using a dimensionless representation of the model's two essential parameters, the scaled slope $s$ of the environmental gradient and the scaled width $c$ of the phenotypic competition function, we showed that most parameter combinations lead to speciation in conjunction with the evolution of short-range dispersal. For shallow gradients, dispersal distance can remain large, precluding speciation. These two types of evolutionary outcome occur in asexual as well as in sexual populations. The third type of evolutionary outcome, occurring at the transition between the two former regimes, qualitatively differs between asexual and sexual populations. In the corresponding parameter regions, asexual populations speciate while evolving long-range dispersal, whereas sexual populations do not speciate while evolving short-range dispersal. A phenotypic gradient following the envi- 
ronmental gradient only arises in the last case, i.e., for non-speciating sexual populations evolving towards short-range dispersal.

Transitions between the three identified evolutionary regimes are typically sharp. This finding is explained by the existence of a positive feedback loop between incipient speciation and evolution of short-range dispersal: short-range dispersal facilitates speciation and speciation promotes short-range dispersal. This feedback not only accounts for the abruptness of transitions between regimes but also for the prevalence of short-range dispersal over a wide range of conditions. When gradients are sufficiently steep, ecological diversification is a better means of expanding an organism's home range than long-range dispersal.

In populations with sexual reproduction, the parameter range over which speciation occurs is smaller than for asexual populations, but still surprisingly large. This illustrates that dispersal evolution can be a powerful alternative means to preference evolution of allowing sexual populations to respond to the frequency-dependent disruptive selection pressures resulting from ecological interactions. The positive feedback between speciation and dispersal evolution contributes to the pertinence of this mechanism.

It has been proposed that clustering along an environmental gradient with impermeable boundaries (Doebeli and Dieckmann 2003) "is driven by edge effects, rather than by frequency-dependent competition” (Polechová and Barton 2005). In agreement with a related investigation of parapatric clustering (Leimar et al. 2008), our analysis here shows that clustering occurs both for impermeable and for cline-periodic boundary conditions. So while impermeable boundaries may affect the details of evolutionary outcomes along environmental gradients- in ways that are worth learning about, since spatial boundaries in nature are real and cannot be conveniently assumed away by theorists - we can conclude that phenotypic clustering in our model occurs independently of boundary effects.

In order not to overload our study with too many parameters, we focused attention on the evolution of natal dispersal distance, as measured by the standard deviation $v$. Several extensions could be worthwhile. For example, one might want to consider the evolution of adult dispersal rates $m$. However, to the extent that dispersal is diffusive, only the compound parameter $\frac{1}{2}(b+m) v^{2}$ matters (Doebeli and Dieckmann 2003), so that the consequences of evolution in $m$ are equivalent to those of evolution in $v$. An analogous compound parameter, $\frac{1}{2} b v^{2}+\frac{1}{2} m \tilde{v}^{2}$, applies when the standard deviation $v$ of natal dispersal distances differs from that of adult dispersal distances, $\tilde{v}$.

Other extensions are expected to be more consequential. In particular, it could be interesting to study costs of dispersal, or spatiotemporal variation in local environmental conditions. These factors can have opposite effects on the evolution of dispersal, and their interaction can lead to complex dynamics. Spatiotemporal variation has been shown to im- 
pact dispersal rates (Van Valen 1971; Travis and Dytham 1998; Travis 2001) as well as dispersal distances (Murrell et al. 2002). Such variation can also lead to dispersal dimorphism, resulting in the coexistence of dispersal morphs with high and low mobility (McPeek and Holt 1992; Johst et al. 1999; Mathias et al. 2001; Parvinen 2002; Heinz and Strand 2006). Travis (2001) found that the evolution of dispersal rates depends not only on whether or not there is temporal or spatial variability in the environment, but also on the form of such variability. Demographic stochasticity (which our model includes) creates spatiotemporal variation in local environmental conditions and has been shown to favor dispersal (Holt and Mc Peek 1996; Cadet et al. 2003; Parvinen et al. 2003), but also to impact the costs of dispersal (Cadet et al. 2003). Increasing costs of dispersal, in the form of dispersal mortality and/or diminished fecundity, naturally favor lower dispersal rates (Poethke and Hovestadt 2002) and shorter dispersal distances (Murell et al. 2002). Yet, when considering kin competition (which our model also includes), the relationship between dispersal mortality and evolved dispersal rate was found to be non-monotonic (Gandon and Michalakis 1999; Gandon 1999; Kisdi 2004). Another interesting extension would be to include the evolution of dispersal directedness. Recent empirical work showed that there is an important link between the directedness of dispersal and the evolution of species: nonrandom dispersal can produce genetic differentiation (Postma and van Noordwijk 2005; Garant et al. 2005) and thereby trigger speciation. The evolution of dispersal directedness in turn has been shown to be dependent on spatial variability (Heinz and Strand 2006) and temporal variability (Armsworth and Roughgarden 2005). One could also consider the population genetics of the phenotypes studied here: for example, Billiard and Lenormand (2005) investigated how linkage between two loci for dispersal and local adaptation affects the evolution of dispersal rates. Finally, it could be interesting to vary the shape of the kernels for competition, dispersal, and mating. In this context, it must be borne in mind - as this sometimes gets overlooked (Polechová and Barton 2005) - that the structural instability of simple deterministic models with normal competition kernels (May and MacArthur 1972; Roughgarden 1974; Sasaki and Ellner 1995; Sasaki 1997; Gyllenberg and Meszéna 2005; Doebeli et al. 2007; Pigolotti et al. 2007) does not apply to the stochastic individualbased models studied here.

Dispersal is subject to evolution and also drives evolutionary dynamics by being an important determinant of the mixing and isolation of populations. It is therefore essential to understand the interaction between dispersal as an evolving character on the one hand and dispersal as an evolutionary factor on the other. Our finding of a positive feedback leading to the combination of short-range dispersal and speciation under a wide range of conditions shows how intricate this interaction can be. We thus hope that this study serves as a first 
440 step towards understanding the complex interplay between dispersal evolution and speci-

441 ation.

442 Acknowledgements

443 We thank Øyvind Fiksen and all members of the modeling group of the Department of Bi-

444 ology at the University of Bergen for fruitful discussions. S.H. gratefully acknowledges

445 financial support by the EU project ETHOFISH (QLRT-2001-00799). R.M. and U.D.

446 gratefully acknowledge financial support by the Vienna Science and Technology Fund 447 (WWTF). 


\section{References}

Abrams PA, Matsuda H, Harada Y (1993) Evolutionarily unstable fitness maxima and stable fitness minima of continuous traits. Evolutionary Ecology 7:465-487

Armsworth PR, Roughgarden JE (2005) Disturbance induces the contrasting evolution of reinforcement and dispersiveness in directed and random movers. Evolution 59:20832096

Bengtsson BO (1978) Avoiding inbreeding: At what cost? Journal of Theoretical Biology 73:439-444

Billiard S, Lenormard T (2005) Evolution of migration under kin selection and local adaptation. Evolution 59:13-23

Bush GL, Smith JJ (1998) The genetics and ecology of sympatric speciation: A case study. Researches on Population Ecology 40:175-187

Cadet C, Ferrière R, Metz JAJ, van Baalen M (2003) The evolution of dispersal under demographic stochasticity. American Naturalist 162:427-441

Christiansen FB, Loeschcke V (1980) Evolution and intraspecific exploitative competition. I. One locus theory for small additive gene effects. Theoretical Population Biology 18:297-313

Day T (2000) Competition and the effect of spatial resource heterogeneity on evolutionary diversification. American Naturalist 155:790-803

Dieckmann U, Doebeli M (1999) On the origin of species by sympatric speciation. Nature 400:354-357

Doebeli M (1996a) A quantitative genetic competition model for sympatric speciation. Journal of Evolutionary Biology 9:893-909

Doebeli M (1996b) An explicit genetic model for ecological character displacement. Ecology $77: 510-520$

Doebeli M, Blok HJ, Leimar O, Dieckmann U (2007) Multimodal pattern formation in phenotype distributions of sexual populations. Proceedings of the Royal Society London Series B 274:347-357

Doebeli M, Dieckmann U (2000) Evolutionary branching and sympatric speciation caused by different types of ecological interactions. American Naturalist 156:S77-S101

Doebeli M, Dieckmann U (2003) Speciation along environmental gradients. Nature 421:259-263 
Doebeli M, Ruxton GD (1997) Evolution of dispersal rates in metapopulation models: Branching and cyclic dynamics in phenotype space. Evolution 51:1730-1741

Drossel B, McKane A (2000) Competitive speciation in quantitative genetic models. Journal of Theoretical Biology 204:467-478

Egas M, Dieckmann U, Sabelis MW (2004) Evolution restricts the coexistence of specialists and generalists: The role of trade-off structure. American Naturalist 163:518-531

Egas M, Sabelis MW, Dieckmann U (2005) Evolution of specialization and ecological character displacement of herbivores along a gradient of plant quality. Evolution 59:507520

Ezoe H (1998) Optimal dispersal range and seed size in a stable environment. Journal of Theoretical Biology 190:287-293

Felsenstein J (1981) Skepticism towards Santa Rosalia, or why are there so few kinds of animals? Evolution 35:124-238

Filchack KE, Roethele JB, Feder JL (2000) Natural selection and sympatric divergence in the apple maggot Rhagoletis pomonella. Nature 407:739-742

Frank SA (1986) Dispersal polymorphisms in subdivided populations. Journal of Theoretical Biology 122:303-309

Gandon S (1999) Kin competition, the cost of inbreeding and the evolution of dispersal. Journal of Theoretical Biology 200:245-364

Gandon S, Michalakis Y (1999) Evolutionarily stable dispersal rate in a metapopulation with extinctions and kin competition. Journal of Theoretical Biology 199:275-290

Garant D, Kruuk LEB, Wilkin TA, McCleery RH, Sheldon BC (2005) Evolution driven by differential dispersal within a wild bird population. Nature 433:60-65

Geritz SAH, Kisdi É, Meszéna G, Metz JAJ (1998) Evolutionarily singular strategies and the adaptive growth and branching of the evolutionary tree. Evolutionary Ecology Research 12:35-57

Geritz SAH, Van der Meijden E, Metz JAJ (1999) Evolutionary dynamics of seed size and seedling competitive ability. Theoretical Population Biology 55:324-343

Grimm V, Berger U, Bastiansen F, Eliassen S, Ginot V, Giske J, Goss-Custard J, Grand T, Heinz S, Huse G, Huth A, Jepsen JU, Jørgensen C, Mooij WM, Müller B, Pe'er G, Piou C, Railsback SF, Robbins AM, Robbins MM, Rossmanith E, Rüger N, Strand E, Souissi S, Stillman RA, Vabø R, Visser U, DeAngelis DL (2006) A standard protocol for describing individual-based and agent-based models. Ecological Modelling 198:115-126 
Grimm V, Railsback SF (2005) Individual-based Modeling and Ecology. Princeton University Press

Grimm V, Revilla E, Berger U, Jeltsch F, Mooij WM, Railsback SF, Thulke HH, Weiner J, Wiegand T, DeAngelis DL (2005) Pattern-oriented modeling of agent-based complex systems: Lessons from ecology. Science 310:987-991

Gyllenberg M, Meszéna G (2005) On the impossibility of coexistence of infinitely many strategies. Journal of Mathematical Biology 50:133-160

Hamilton WD, May RM (1977) Dispersal in stable habitats. Nature 269:578-581

Heinz SK, Strand E (2006) Adaptive patch searching strategies in fragmented landscapes. Evolutionary Ecology 20:113-130

Higashi M, Takimoto G, Yamamura N (1999) Sympatric speciation by sexual selection. Nature 402:523-526

Holt RD, McPeek MA (1996) Chaotic population dynamics favors the evolution of dispersal. American Naturalist 148:709-718

Hovestadt T, Messner S, Poethke HJ (2001) Evolution of reduced dispersal mortality and 'fat-tailed' dispersal kernels in autocorrelated landscapes. Proceedings of the Royal Society of London Series B 268:385-391

Ito HC, Dieckmann U (2007) A new mechanism for recurrent adaptive radiations. American Naturalist 170:E96-E111

Jansen VAA, Mulder GSEE (1999) Evolving biodiversity. Ecology Letters 2:379-386

Johst K, Doebeli M, Brandl R (1999) Evolution of complex dynamics in spatially structured populations. Proceedings of the Royal Society of London B 266:1147-1154

Kawecki TJ (1997) Sympatric speciation via habitat specialization driven by deleterious mutations. Evolution 51:1751-1763

Kisdi É (1999) Evolutionary branching under asymmetric competition. Journal of Theoretical Biology 197:149-162

Kisdi É (2001) Long-term adaptive diversity in Levene-type models. Evolutionary Ecology Research 3:721-727

Kisdi É (2004) Conditional dispersal under kin competition: Extension of the HamiltonMay model to brood size-dependent dispersal. Theoretical Population Biology 66:369380

Kisdi É, Geritz SAH (2001) Evolutionary disarmament in interspecific competition. Proceedings of the Royal Society of London B 268:2589-2594 
Kisdi É, Jacobs FJA, Geritz SAH (2001) Red Queen evolution by cycles of evolutionary branching and extinction. Selection 2:161-176

Kondrashov AS, Kondrashov F (1999) Interactions among quantitative traits in the course of sympatric speciation. Nature 400:351-354

Law R, Marrow P, Dieckmann U (1997) On evolution under asymmetric competition. Evolutionary Ecology 11:485-501

Leimar O, Doebeli M, Dieckmann U (2008) Evolution of phenotypic clusters through competition and local adaptation along an environmental gradient. Evolution, in press

MacArthur RH, Levins R (1967) The limiting similarity, convergence, and divergence of coexisting species. American Naturalist 101:377-385

Mathias A, Kisdi É, Olivieri I (2001) Divergent evolution of dispersal in a heterogeneous landscape. Evolution 55:246-259

May RM, MacArthur RH (1972) Niche overlap as a function of environmental variability. Proceedings of the National Academy of Science of the USA 19:1109-1113

Maynard Smith J (1966) Sympatric speciation. American Naturalist 100:637-650

Maynard Smith J, Brown RL (1986) Competition and body size. Theoretical Population Biology 30:166-179

McPeek MA, Holt RD (1992) The evolution of dispersal in spatially and temporally varying environments. American Naturalist 140:1010-1027

Meszéna G, Czibula I, Geritz SAH (1997) Adaptive dynamics in a 2-patch environment: A toy model for allopatric and parapatric speciation. Journal of Biological Systems 5:265284

Metz JAJ, Geritz SAH, Meszéna G, Jacobs FJA, van Heerwaarden JS (1996) Adaptive dynamics: A geometrical study of the consequences of nearly faithful reproduction. In Stochastic and Spatial Structures of Dynamical Systems, Proceedings of the Royal Dutch Academy of Science (KNAW Verhandelingen), eds. van Strien SJ, Verduyn Lunel SM, pp. 183-231. Dordrecht, Netherlands:North Holland

Mizera F, Meszéna G (2003) Spatial niche packing, character displacement and adaptive speciation along an environmental gradient. Evolutionary Ecology Research 5:363-382

Motro U (1991) Avoiding inbreeding and sibling competition: The evolution of sexual dimorphism for dispersal. American Naturalist 137:108-115

Murrell DJ, Dytham C, Travis JMJ (2002) The evolution of dispersal distance: Consequences for population dynamics. Oikos 97:229-236 
Olivieri I, Michalakis Y, Gouyon PH (1995) Metapopulation genetics and the evolution of dispersal. American Naturalist 146:202-228

Parvinen K (2002) Evolutionary branching of dispersal strategies in structured metapopulations. Journal of Mathematical Biology 45:106-124

Parvinen K, Dieckmann U, Gyllenberg M, Metz JAJ (2003) Evolution of dispersal in metapopulations with local density dependence and demographic stochasticity. Journal of Evolutionary Biology 16:143-153

Pigolotti S, López C, Hernández-García E (2007) Species clustering in competitive LotkaVolterra models. Physical Review Letters 98:258101

Poethke HJ, Hovestadt T (2002) Evolution of density and patch-size dependent dispersal rates. Proceedings of the Royal Society of London Series B 269:637-645

Polechová J, Barton NH (2005) Speciation through competition: A critical review. Evolution 59:1194-1210

Postma E, van Noordwijk AJ (2005) Gene flow maintains a large genetic difference in clutch size at a small spatial scale. Nature 433:65-68

Ronce O, Brachet B, Olivieri I, Gouyon PH, Clobert J (2005) Plastic changes in seed dispersal along ecological succession: Theoretical predictions from an evolutionary model. Journal of Ecology 93:431-440

Ronce O, Perret F, Olivieri I (2000) Evolutionary stable dispersal rates do not always increase with local extinction rates. American Naturalist 155:485-496

Rosenzweig ML (1978) Competitive speciation. Biological Journal of the Linnean Society 10:275-289

Roughgarden J (1974) Species packing and the competition function with illustrations from coral reef fish. Theoretical Population Biology 5:163-186

Rousset F, Gandon S (2002) Evolution of the distribution of dispersal distance under distance-dependent cost of dispersal. Journal of Evolutionary Biology 15:515-523

Sasaki A (1997) Clumped distribution by neighborhood competition. Journal of Theoretical Biology 186:415-430

Sasaki A, Ellner S (1995) The evolutionarily stable phenotype distribution in a random environment. Evolution 40:337-350

Savill NJ, Hogeweg P (1998) Spatially induced speciation prevents extinction: The evolution of dispersal distance in oscillatory predator-prey systems. Proceedings of the Royal Society of London Series B 265:25-32 
Schliewen U, Rassmann K, Markmann M, Markert J, Kocher T, Tautz D (2001) Genetic and ecological divergence of a monophyletic cichlid species pair under fully sympatric conditions in Lake Ejagham, Cameroon. Molecular Ecology 10:1471-1488

Schliewen UK, Tautz D, Pääbo S (1994) Sympatric speciation suggested by monophyly of crater lake cichlids. Nature 368:629-632

Schreiber SJ, Tobiason GA (2003) The evolution of resource use. Journal of Mathematical Biology 47:56-78

Seger J (1985) Intraspecific resource competition as a cause of sympatric speciation. In Evolution: Essays in Honour of John Maynard Smith, eds. Greenwood PJ, Harvey PH, Slatkin M, pp. 43-53. Cambridge, UK: Cambridge University Press

Slatkin M (1973) Gene flow and selection in a cline. Genetics 75:733-756

Slatkin M (1980) Ecological character displacement. Ecology 61:163-177

Taper ML, Case TJ (1985) Quantitative genetic models for the coevolution of character displacement. Ecology 66:355-371

Taylor PD (1988) An inclusive fitness model for dispersal of offspring,. Journal of Theoretical Biology 130:363-378

Travis JMJ (2001) The color of noise and the evolution of dispersal. Ecological Research 16:157-163

Travis JMJ, Dytham C (1998) The evolution of dispersal in a metapopulation: A spatially explicit, individual-based model. Proceedings of the Royal Society London B 265:17-23

Turelli M, Barton NH, Coyne JA (2001) Theory and speciation. Trends in Ecology and Evolution 16:330-343

Turesson G (1922) The genotypical response of the plant species to the habitat. Hereditas 3:211-350

Turner GF, Burrows MT (1995) A model of sympatric speciation by sexual selection. Proceedings of the Royal Society of London B 260:287-292

Van Valen L (1971) Group selection and the evolution of dispersal. Evolution 25:591-598

Via S (2001) Sympatric speciation in animals: The ugly duckling grows up. Trends in Ecology and Evolution 16:381-390

Vincent TL, Cohen Y, Brown JS (1993) Evolution via strategy dynamics. Theoretical Population Biology 44:149-176

Waser NM (1986) Flower constancy: Definition, cause, and measurement. American Naturalist 127:593-603 
645 Wilson AB, Noack K, Meyer A (2000) Incipient speciation in sympatric Nicaraguan crater 646 lake cichlid fishes: Sexual selection vs. ecological diversification. Proceedings of the 647 Royal Society of London Series B 267:2133-2141

648 Wright S (1943) Isolation by distance. Genetics 28:114-138 
Table 1. Overview of parameters and their default values.

Maximal carrying capacity density, scaling the number of individuals $\quad K_{0}$

300

in the system

Standard deviation of carrying capacity density, scaling the reduction

$\sigma_{K}$

0.3

of carrying capacity density with increased phenotypic distance in the ecological character from the locally optimal phenotype

Standard deviation of phenotypic competition function, scaling the re-

$\sigma_{c} \quad$ variable duction of competition strength with increased phenotypic distance in the ecological character between individuals

Standard deviation of spatial competition function, scaling the reduc$\sigma_{s} \quad 0.2$ tion of competition strength with increased spatial distance between individuals

Slope of environmental gradient $g$ variable

Per capita birth rate, scaling time b $\quad 1$

Mutation probability

$\mu_{m} \quad 0.02$

Standard deviation of distribution of mutation effects

$\sigma_{m} \quad 0.01$

Scaled standard deviation of phenotypic competition function

c $\quad \sigma_{c} / \sigma_{K}$

Scaled slope of environmental gradient

$S \quad g \sigma_{s} / \sigma_{K}$

Table 2. Overview of evolutionary outcomes.

\begin{tabular}{|l|l|l|l|}
\hline \multicolumn{2}{|c|}{ Asexual populations } & \multicolumn{2}{c|}{ Sexual populations } \\
\hline Steep slope & $\begin{array}{l}\text { Speciation, } \\
\text { short-range dispersal }\end{array}$ & $\begin{array}{l}\text { Steep slope, } \\
\text { wide competition }\end{array}$ & $\begin{array}{l}\text { Speciation, } \\
\text { short-range dispersal }\end{array}$ \\
\hline $\begin{array}{l}\text { Shallower slope, } \\
\text { narrow competition }\end{array}$ & $\begin{array}{l}\text { Speciation, } \\
\text { long-range dispersal }\end{array}$ & $\begin{array}{l}\text { Steep slope, } \\
\text { narrow competition }\end{array}$ & $\begin{array}{l}\text { No speciation, } \\
\text { short-range dispersal }\end{array}$ \\
\hline $\begin{array}{l}\text { Shallower slope, } \\
\text { wide competition }\end{array}$ & $\begin{array}{l}\text { No speciation, } \\
\text { long-range dispersal }\end{array}$ & Shallower slope & $\begin{array}{l}\text { No speciation, } \\
\text { long-range dispersal }\end{array}$ \\
\hline
\end{tabular}


Figure 1. Outcomes of asexual evolution when boundaries in the $x$-direction are impermeable. (a) Dependence of outcomes on the model's two dimensionless parameters $s$ (scaled slope of environmental gradient) and $c$ (scaled width of phenotypic competition function). Three types of outcomes can occur, resulting in three sharply delineated regions in parameter space: (i) speciation and short-range dispersal (right-hand side), (ii) speciation and longrange dispersal (lower left corner), and (iii) no speciation and long-range dispersal (upper left corner). (b) Dependence of scaled dispersal distances on $s$, for three different values of $c$. The standard deviation of evolved distances is indicated by the gray areas. Decreasing the slope results in the sharp rise of scaled dispersal distance for $c=2$ and $c=1$, while this transition is less abrupt for $c=0.5$. Other parameters as shown in Table 1 .

Figure 2. Outcomes of asexual evolution when boundaries in the $x$-direction are clineperiodic. (a) Dependence of outcomes on the model's two dimensionless parameters $s$ (scaled slope of environmental gradient) and $c$ (scaled width of phenotypic competition function). (b) Dependence of scaled dispersal distances on $s$, for three different values of c. Other details as in Fig. 1.

Figure 3. Outcomes of sexual evolution when boundaries in the $x$-direction are impermeable. (a) Dependence of outcomes on the model's two dimensionless parameters $s$ (scaled slope of environmental gradient) and $c$ (scaled width of phenotypic competition function). Three types of outcomes can occur, resulting in three sharply delineated regions in parameter space: (i) speciation and short-range dispersal (upper right corner), (ii) no speciation and short-range dispersal (lower right corner), and (iii) no speciation and long-range dispersal (left-hand side). (b) Dependence of scaled dispersal and mating distances on $s$, for three different values of $c$. The standard deviations of evolved distances are indicated by the gray areas and thin continuous curves, respectively. Decreasing the slope results in the sharp rise of scaled dispersal distance for $c=2, c=1$, and $c=0.5$. Other parameters as shown in Table 1.

Figure 4. Outcomes of sexual evolution when boundaries in the $x$-direction are clineperiodic. (a) Dependence of outcomes on the model's two dimensionless parameters $s$ (scaled slope of environmental gradient) and $c$ (scaled width of phenotypic competition function). (b) Dependence of scaled dispersal and mating distances on $s$, for three different values of $c$. Other details as in Fig. 3.

Figure 5. Evolution of phenotypic and spatial distributions when boundaries in the $x$ direction are impermeable. Three examples each are shown for asexual populations (left 
684 column) and sexual populations (right column). The left panel of each example shows the 685 evolution of the distribution of the ecological character in the range $0 \leq u \leq 1$ from time 0 686 (bottom) to time 15,000 (top). The corresponding right panel shows the final spatial distri687 bution of individuals at time 15,000 with different grayscales depicting different values of 688 the ecological character. Asexual populations: (a) speciation with short-range dispersal 689 ( $s=0.66, c=1.33$ ), (b) speciation with long-range dispersal ( $s=0.16, c=0.47$ ), and (c) 690 speciation with short-range dispersal $(s=0.10, c=1.53)$. Sexual populations: (d) speci691 ation with short-range dispersal ( $s=0.83, c=1.66$ ), (e) no speciation with short-range 692 dispersal ( $s=0.80, c=0.33$ ), and (f) no speciation with long-range dispersal ( $s=0.13$, $693 \quad c=0.80)$. The examples illustrate that short-range dispersal induces spatial structure and 694 facilitates speciation. 


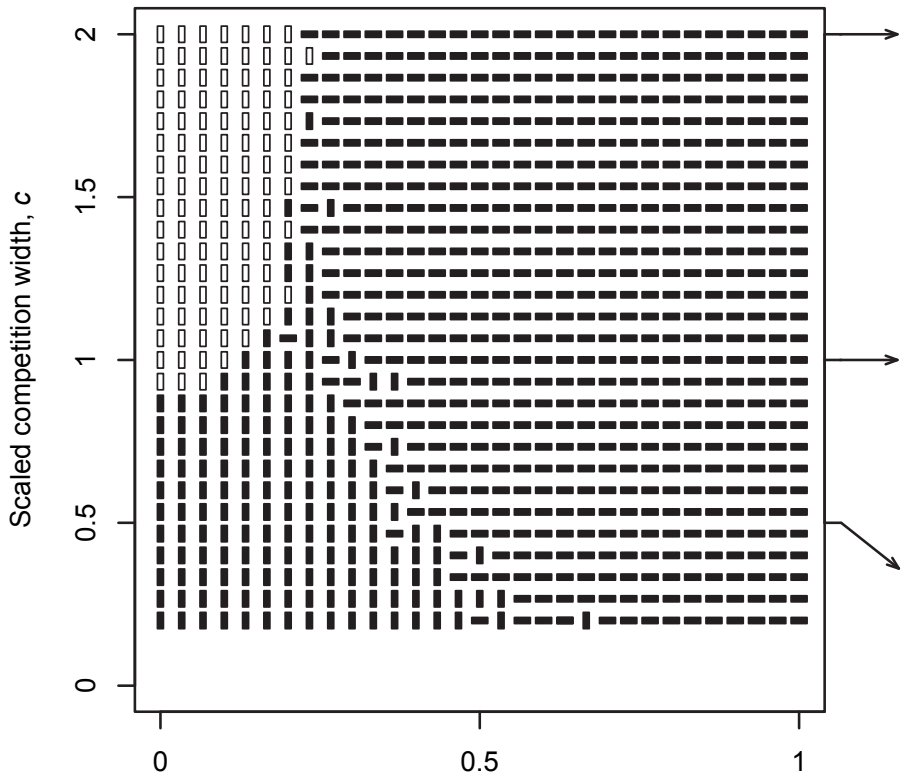

Scaled slope of gradient, $s$

- Speciation, short-range dispersal

- Speciation, long-range dispersal

0. No speciation, long-range dispersal

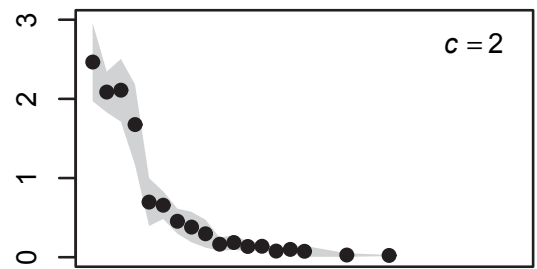

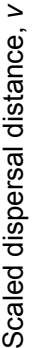
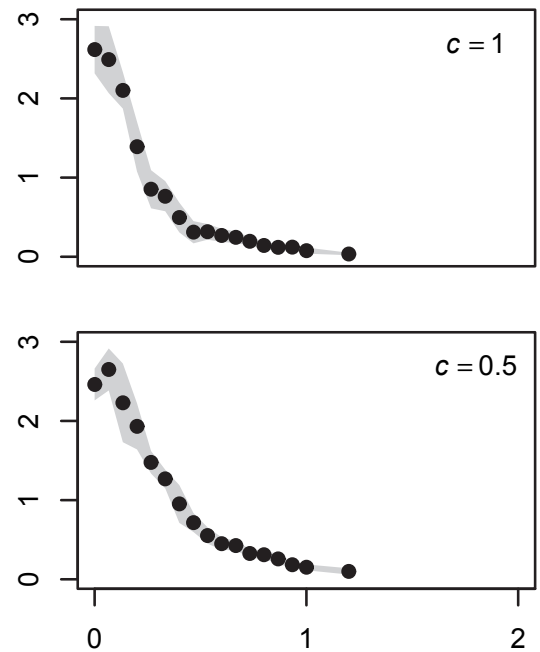

Scaled slope of gradient, $s$ 


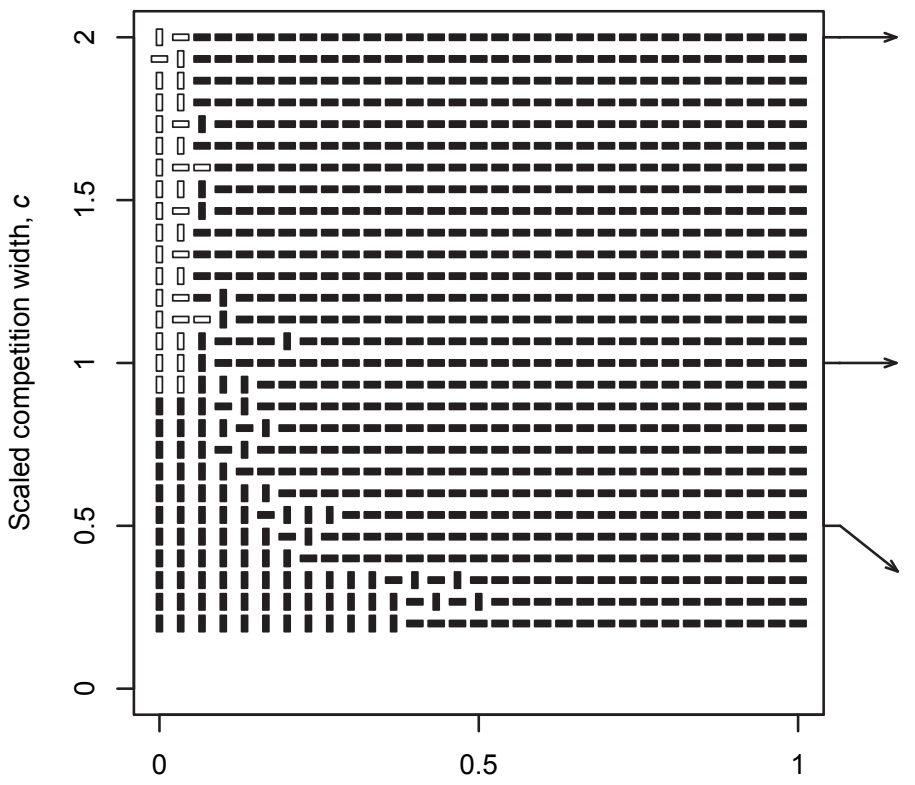

Scaled slope of gradient, $s$

- Speciation, short-range dispersal

- Speciation, long-range dispersal

(1) No speciation, long-range dispersal

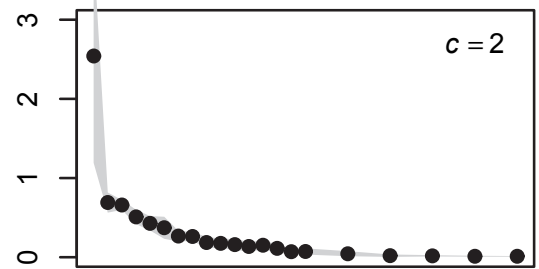

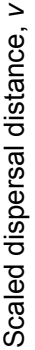
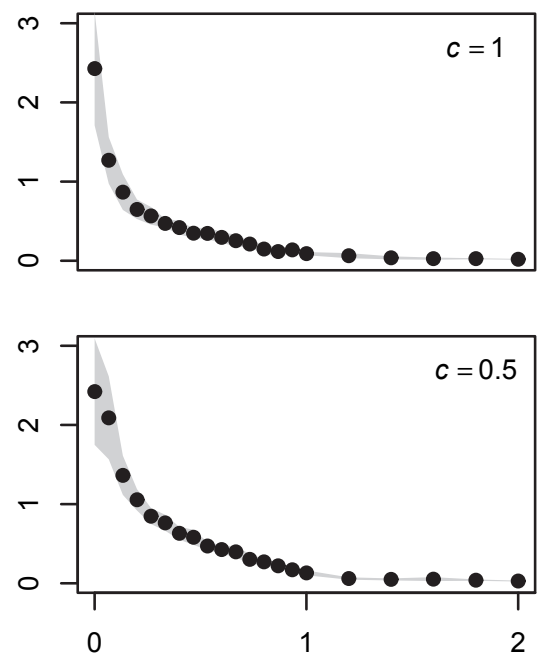

Scaled slope of gradient, $s$ 


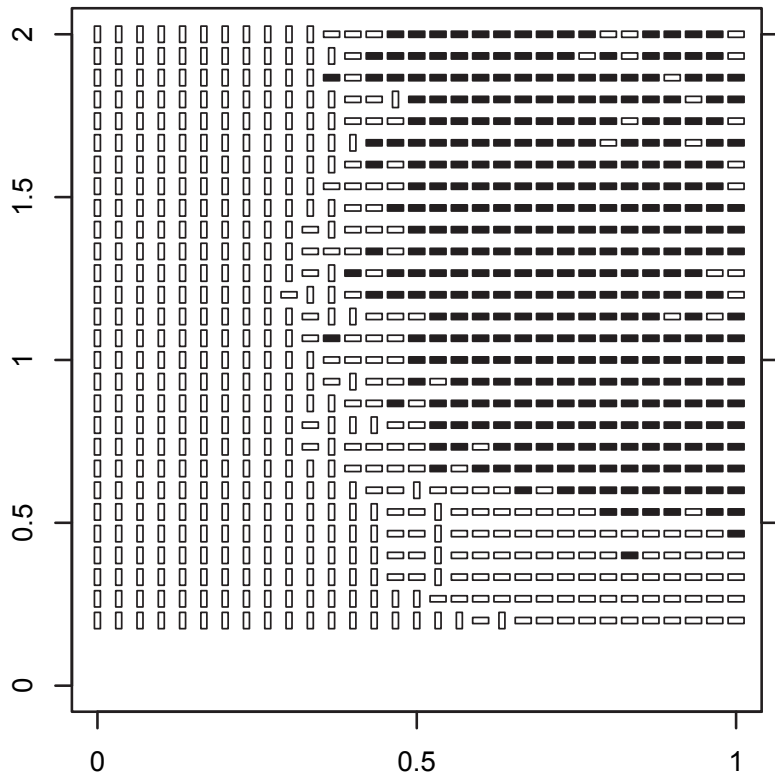

Scaled slope of gradient, $s$

- Speciation, short-range dispersal

- No speciation, short-range dispersal

0 No speciation, long-range dispersal
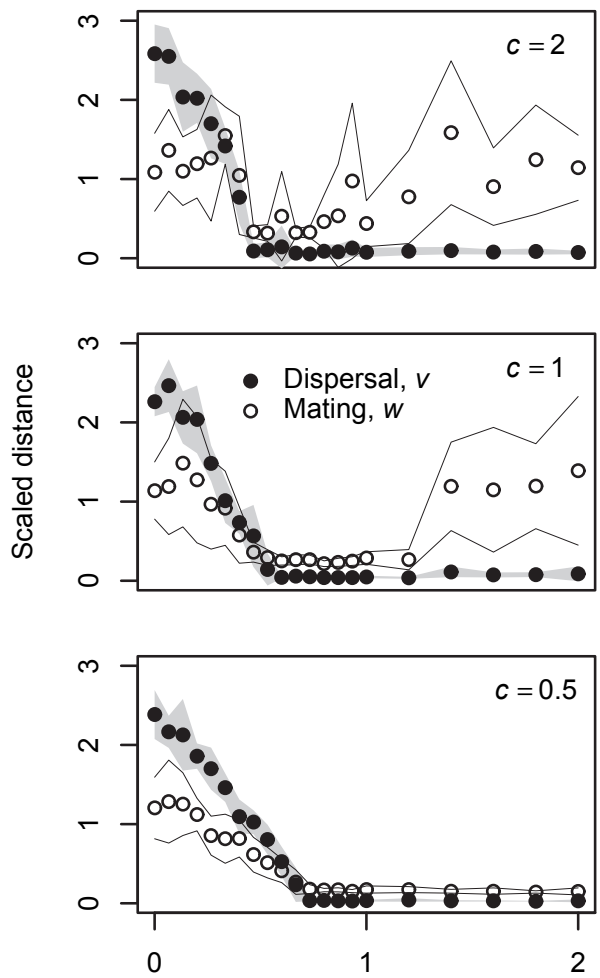

Scaled slope of gradient, $s$ 


\section{Asexual populations}

a
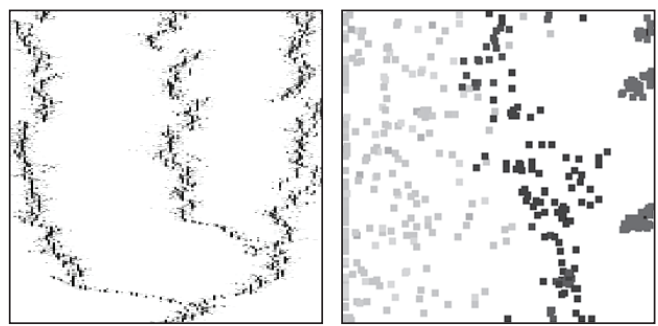

b
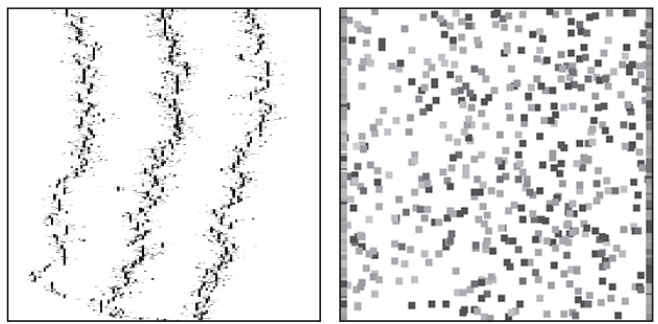

C
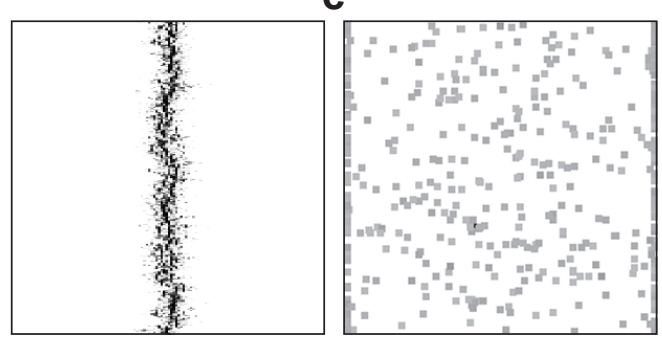

Sexual populations
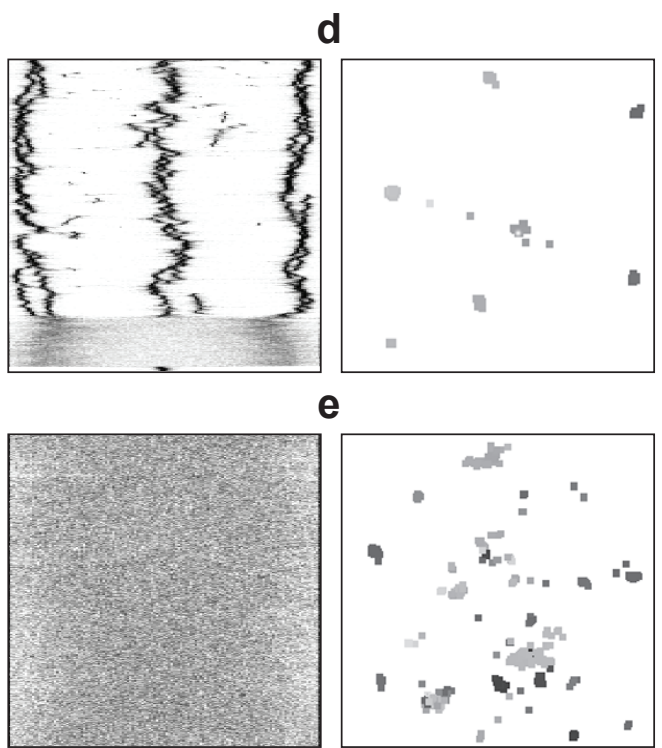

f

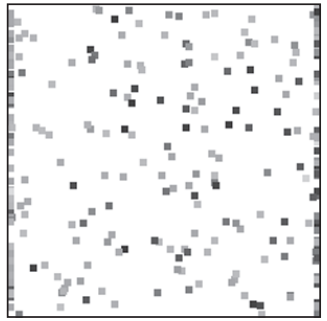

